\section{INTERRUPTS: An interrupt routine for Apple II PASCAL 1.1}

\author{
CHRIS IDZIKOWSKI \\ M.R.C. Applied Psychology Unit, Cambridge CB2 2EF, England
}

Normally, when a nonmaskable interrupt occurs on an Apple 6502, the microprocessor responds by performing some operations on its stack (push program counter high byte, push program counter low byte, and push status register) and performing an indirect jump via hexadecimal FFFB (high byte) and FFFA (low byte). When PASCAL is not in use, these locations are in ROM and contain the hexadecimal location 03FB; the microprocessor continues executing what it finds there. When PASCAL is operating, the language card is more likely to be in use than ROM and it holds the same addresses that are used by the "reset" routines. Consequently, if any interrupt occurs during the execution of a PASCAL program, the response is likely to be a rebooting of the system.

The solution employed in INTERRUPTS is simply to rewrite the addresses at the top of RAM to suit the convenience of the experimenter. The program replaces the original addresses after execution of the program if this is what is desired. The interrupt codes that are referenced at either hexadecimal FFFE/FFFF (maskable interrupts) or hexadecimal FFFA/FFFB (nonmaskable interrupts) reside in the PASCAL data heap and are thus under the control of the initiating program. Apart from resolving these references and loading the interrupt routines themselves, the program can also set up data

This research was supported by the Medical Research Council's Army Personnel Research Committee. I am grateful for useful discussions with Ray Bloomfield and Pete Cresswell. areas that may be referenced by the assembly routines; thus, for example, an interrupt routine could read the time from a clock and store this datum in an area that the PASCAL program could use.

Implementation. The interrupt routines are assembled using the PASCAL assembler, so the assembler's features may be utilized. All of the program's procedures and functions can be referenced by the user. At its simplest, if a user wants an interrupt serviced and the internupt does not have to interact with the PASCAL system, then a single call to the program, with the statement "Uses INTERRUPTS;" is all that is required. Since the I/O drivers on the Apple are not interrupt driven, external interrupts can hamper disk transfers, so attempts to transfer data to disk should be avoided if interrupts are operating. However, the INTERRUPTS program's data storage procedures can be used, and the data can be transferred when convenient.

The INTERRUPTS program has been successfully used in a number of experiments. One of these included an animated visual display and measurement of reaction times. The visual display was driven by nonmaskable interrupts, which in turn were driven by an Intel 8523 clock. The subject's push-button response to various features of the display generated maskable interrupts (the servicing routine for this read the time, stored it, and also signaled the PASCAL program to change the display). This unit has also been found useful in biofeedback experiments, in which nonmaskable interrupts are used to control sampling from an A/D board, which in turn controls feedback via the high-resolution screen to the subject. Simple reaction time is simultaneously measured using maskable interrupts.

Availability. The unit is available from the author both as a listing and, if a floppy is provided, on disk.

(Accepted for publication July 10, 1982.) 